PROCEEDINGS OF THE

AMERICAN MATHEMATICAL SOCIETY

Volume 129, Number 11, Pages 3331-3338

S 0002-9939(01)05917-2

Article electronically published on March 29, 2001

\title{
ON THE BEREZIN-TOEPLITZ CALCULUS
}

\author{
L. A. COBURN
}

(Communicated by Joseph A. Ball)

\begin{abstract}
We consider the problem of composing Berezin-Toeplitz operators on the Hilbert space of Gaussian square-integrable entire functions on complex $n$-space, $\mathbf{C}^{n}$. For several interesting algebras of functions on $\mathbf{C}^{n}$, we have $T_{\varphi} T_{\psi}=T_{\varphi \diamond \psi}$ for all $\varphi, \psi$ in the algebra, where $T_{\varphi}$ is the Berezin-Toeplitz operator associated with $\varphi$ and $\varphi \diamond \psi$ is a "twisted" associative product on the algebra of functions. On the other hand, there is a $C^{\infty}$ function $\varphi$ for which $T_{\varphi}$ is bounded but $T_{\varphi} T_{\varphi} \neq T_{\psi}$ for any $\psi$.
\end{abstract}

\section{INTRODUCTION}

For $z=\left(z_{1}, \ldots, z_{n}\right)$ in complex $n$-space, $\mathbf{C}^{n}$, with $z_{j}$ in $\mathbf{C}, z \cdot w=z_{1} \overline{w_{1}}+\ldots+$ $z_{n} \bar{w}_{n}$, consider the space $L^{2}\left(\mathbf{C}^{n}, d \mu\right)$ of Gaussian square-integrable complex-valued functions on $\mathbf{C}^{n}$, with $d \mu(z)=\exp \left\{-|z|^{2} / 2\right\} d v(z)(2 \pi)^{-n}$ with $d v(z)$ Lebesgue measure. The entire functions in $L^{2}\left(\mathbf{C}^{n}, d \mu\right)$ form a closed subspace $H^{2}\left(\mathbf{C}^{n}, d \mu\right)$ which arises naturally as a representation space of the Heisenberg group $[\mathrm{B}],[\mathrm{F}]$, [BC1], C]. On this (Segal-Bargmann) space, there are natural operators, formally introduced by Berezin $\left[\mathrm{Be}\right.$, defined densely for $\varphi(\cdot)$ with $\varphi(w) e^{w \cdot a}$ in $L^{2}\left(\mathbf{C}^{n}, d \mu\right)$ for all $a$ in $\mathbf{C}^{n}$, by

$$
\left(T_{\varphi} f\right)(z)=\int_{\mathbf{C}^{n}} e^{z \cdot w / 2} \varphi(w) f(w) d \mu(w) .
$$

The (possibly unbounded) operator $T_{\varphi}$ is called the Berezin-Toeplitz operator associated to $\varphi$. Note that $H^{2}\left(\mathbf{C}^{n}, d \mu\right)$ is a Bergman space with reproducing kernel function $e^{z \cdot a / 2}$ for the functional of "evaluation at $a$ " $\left[\mathrm{B}\right.$. Note also that $T_{\varphi}=0$ if and only if $\varphi=0[\mathrm{~F}$, p. 140].

The operators $T_{\varphi}$ are closely related to pseudodifferential operators on $L^{2}\left(\mathbf{R}^{n}, d v\right)$. For $\varphi$ bounded, and somewhat more generally, the relation is given by

$$
B^{-1} T_{\varphi} B=W_{\beta_{\varphi}}
$$

where $B$ is the Bargmann isometry $\mathrm{Gu}], W_{\beta}$ is the Weyl operator on $L^{2}\left(\mathbf{R}^{n}, d v\right)$ given by

$$
\left(W_{\beta} g\right)(x)=(2 \pi)^{-n} \int_{\mathbf{R}^{n}} \int_{\mathbf{R}^{n}} \beta\left(\xi, \frac{x+y}{2}\right) e^{i(x-y) \cdot \xi} g(y) d y d \xi
$$

Received by the editors December 21, 1999 and, in revised form, March 21, 2000.

2000 Mathematics Subject Classification. Primary 47B35; Secondary 47B32.

The author's research was supported by a grant of the NSF and a visiting membership in the Erwin Schrödinger Institute. 
and

$$
\beta_{\varphi}(\xi, x)=\pi^{-n} \int_{\mathbf{C}^{n}} \varphi(w) e^{-|w-(x-i \xi)|^{2}} d v(w) .
$$

The operators $T_{\varphi}$ might, therefore, be expected to share many of the properties of pseudodifferential operators. It is not easy to demonstrate a complete equivalence, partly because $\beta_{\varphi}$ is a "very smoothed" version of $\varphi$. The analytic structure of $H^{2}\left(\mathbf{C}^{n}, d \mu\right)$ also enters the picture so that, for example,

$$
T_{\varphi} T_{z_{j}}=T_{\varphi z_{j}} .
$$

Moreover, the available function-theoretic machinery on $H^{2}\left(\mathbf{C}^{n}, d \mu\right)$ is relatively rudimentary, limited primarily to the Bergman space structure and the structure inherited as a representation space of the Heisenberg group.

In this note, we deal with the composition problem: is there a function $\varphi \diamond \psi$ so that

$$
T_{\varphi} T_{\psi}=T_{\varphi \diamond \psi} ?
$$

As a consequence of representation-theoretic results in [C], we do have $(*)$ for a reasonably large class of bounded $\varphi, \psi$ and there is an explicit formula for $\varphi \diamond \psi$. The same "Moyal-type" formula also holds for a large class of unbounded $\varphi, \psi$ (with unbounded $T_{\varphi}, T_{\psi}, T_{\varphi \diamond \psi}$ ) - precisely, $\varphi, \psi$ can be arbitrary polynomials in $\left\{z_{j}, \bar{z}_{j}: 1 \leq j \leq n\right\}$.

On the other hand, we will exhibit a $\varphi$ (unbounded, but $C^{\infty}$ ), for which $T_{\varphi}$ is a bounded operator but $T_{\varphi} T_{\varphi}$ cannot be approximated in norm by bounded BerezinToeplitz operators. Thus, there is a genuine limitation on our ability to compose Berezin-Toeplitz operators.

I thank Samuel D. Schack for useful comments.

\section{Composition of Berezin-Toeplitz operators}

For $C^{\infty}$ functions $\varphi, \psi$ we consider the (formal) twisted product

$$
\varphi \diamond \psi=\sum_{k} \frac{(-2)^{|k|}}{k !}\left(\partial^{k} \varphi\right)\left(\bar{\partial}^{k} \psi\right)
$$

where $k=\left(k_{1}, \ldots, k_{n}\right)$ with $k_{j}$ non-negative integers, and

$$
\begin{aligned}
\partial_{j} & =\frac{\partial}{\partial z_{j}}, \bar{\partial}_{j}=\frac{\partial}{\partial \bar{z}_{j}}, \\
\partial^{k} & =\partial_{1}^{k_{1}} \ldots \partial_{n}^{k_{n}}, \bar{\partial}^{k}=\bar{\partial}_{1}^{k_{1}} \ldots \bar{\partial}_{n}^{k_{n}}, \\
|k| & =k_{1}+k_{2}+\ldots+k_{n}, \\
k ! & =k_{1} ! k_{2} ! \ldots k_{n} !
\end{aligned}
$$

In the cases we will consider, the sum in $(* *)$ will converge.

The first case we consider arises from representation-theoretic considerations of the Heisenberg group [C]. We consider $\varphi, \psi$ in the "smooth Bochner algebra" $B_{a}\left(\mathbf{C}^{n}\right)$ which consists of all Fourier-Stieltjes transforms of compactly supported, regular, bounded complex-valued Borel measures on $\mathbf{C}^{n}$. More precisely, let

$$
\chi_{a}(z)=\exp \{i \operatorname{Im}(z \cdot a)\} .
$$

Then $B_{a}\left(\mathbf{C}^{n}\right)$ consists of all functions

$$
\hat{\sigma}(z)=\int_{\mathbf{C}^{n}} \chi_{a}(z) d \sigma(a)
$$


where $\sigma$ is a compactly supported, regular, bounded complex-valued Borel measure. It is well known that such functions are bounded, uniformly continuous, with bounded derivatives of all orders.

As our first positive result, we have

Theorem 1. For $\varphi, \psi$ in $B_{a}\left(\mathbf{C}^{n}\right), \varphi \diamond \psi$ is also in $B_{a}\left(\mathbf{C}^{n}\right)$ and $T_{\varphi} T_{\psi}=T_{\varphi \diamond \psi}$. The series in $(* *)$ converges uniformly and absolutely.

Proof. In [C], it was shown that for $\varphi=\hat{\sigma}, \psi=\hat{\tau}$ in $B_{a}\left(\mathbf{C}^{n}\right)$,

$$
T_{\varphi} T_{\psi}=T_{(\sigma \diamond \tau)^{\wedge}} .
$$

Here, we defined $\sigma \diamond \tau$ for all $\phi$ in $C_{0}\left(\mathbf{C}^{n}\right)$ by

$$
\int_{\mathbf{C}^{n}} \phi(c) d(\sigma \diamond \tau)(c)=\int_{\mathbf{C}^{n}} \int_{\mathbf{C}^{n}} \phi(a+b) e^{b \cdot a / 2} d \sigma(a) d \tau(b)
$$

so that

$$
(* * *) \quad(\sigma \diamond \tau)^{\Upsilon}(z)=\int_{\mathbf{C}^{n}} \int_{\mathbf{C}^{n}} \chi_{a+b}(z) e^{b \cdot a / 2} d \sigma(a) d \tau(b)
$$

is in $B_{a}\left(\mathbf{C}^{n}\right)$.

Expanding $e^{b \cdot a / 2}$ in MacLaurin series in $(* * *)$ gives

$$
\begin{aligned}
(\sigma \diamond \tau)^{\uparrow}(z) & =\sum_{s=0}^{\infty} \sum \frac{1}{s !} \frac{1}{2^{s}} \sum_{1 \leq j_{i} \leq n} \int \bar{a}_{j_{1}} \ldots \bar{a}_{j_{s}} \chi_{a}(z) d \sigma(a) \int b_{j_{1}} \ldots b_{j_{s}} \chi_{b}(z) d \tau(b) \\
& =\sum_{s=0}^{\infty} \frac{1}{s !} \frac{1}{2^{s}} \sum_{1 \leq j_{i} \leq n} 2^{s}\left(\partial_{j_{1}} \ldots \partial_{j_{s}} \varphi\right)(-2)^{s}\left(\bar{\partial}_{j_{1}} \ldots \bar{\partial}_{j_{s}} \psi\right) \\
& =\sum_{s=0}^{\infty} \frac{(-2)^{s}}{s !} \sum_{1 \leq j_{i} \leq n}\left(\partial_{j_{1}} \ldots \partial_{j_{s}} \varphi\right)\left(\bar{\partial}_{j_{1}} \ldots \bar{\partial}_{j_{s}} \psi\right) \\
& =\sum_{k} \frac{(-2)^{|k|}}{k !}\left(\partial^{k} \varphi\right)\left(\bar{\partial}^{k} \psi\right)
\end{aligned}
$$

and it is clear that the series converges uniformly and absolutely. Comparison with $(* *)$ shows that

$$
T_{\varphi} T_{\psi}=T_{\varphi \diamond \psi}
$$

and completes the proof.

Our second case consists of $\varphi, \psi$ arbitrary polynomials in $\left\{z_{j}, \bar{z}_{j}: 1 \leq j \leq n\right\}$. Here, the operators $T_{\varphi}, T_{\psi}$ are unbounded and we need to be a little more careful. Nevertheless, we have for $\varphi \diamond \psi$ given by $(* *)$,

Theorem 2. For $\varphi, \psi$ polynomials in $\left(z_{1}, \ldots, z_{n}, \bar{z}_{1}, \ldots, \bar{z}_{n}\right)$, we have $T_{\varphi} T_{\psi}$ defined on a dense domain consisting of linear combinations of functions of the form $\left\{p(z) e^{z \cdot a}: a \in \mathbf{C}^{n}\right.$ and $p(z)$ polynomial in $\left.\left(z_{1}, \ldots, z_{n}\right)\right\}$. On this domain

$$
T_{\varphi} T_{\psi}=T_{\varphi \diamond \psi}
$$

and $\varphi \diamond \psi$ is polynomial in the $z_{j}, \bar{z}_{j}$. 
Proof. Clearly, $T_{\bar{z}_{j}}=2 \partial_{j}$ and it is now easy to check that $T_{\varphi} p(z) e^{z \cdot a}=q(z) e^{z \cdot a}$ where $p, q$ are polynomial in $z_{1}, \ldots, z_{n}$. The proof of the composition formula is inductive, in several steps.

We note first that, for $\varphi$ polynomial in $\left\{z_{j}, \bar{z}_{j}: 1 \leq j \leq n\right\}, T_{\varphi} T_{\bar{z}_{j}}=T_{\varphi \diamond \bar{z}_{j}}$ implies $T_{\varphi \diamond\left|z_{j}\right|^{2}}=T_{\varphi} T_{\left|z_{j}\right|^{2}}$. This is because

$$
\begin{aligned}
T_{\varphi} T_{\left|z_{j}\right|^{2}} & =\left(T_{\varphi} T_{\bar{z}_{j}}\right) T_{z_{j}} \\
& =T_{\left(\varphi \diamond \bar{z}_{j}\right) z_{j}}=T_{\varphi \diamond\left|z_{j}\right|^{2}} .
\end{aligned}
$$

Next, we check inductively that $T_{\varphi} T_{\bar{z}_{j}}=T_{\varphi \diamond \bar{z}_{j}}$ for all $\varphi$ polynomial in $\left\{z_{j}, \bar{z}_{j}: 1 \leq\right.$ $j \leq n\}$. It is enough to consider $\varphi$ monomial. Assume the result for $\varphi$ of fixed degree ( $\varphi$ constant is trivial). The inductive step is:

$$
\begin{aligned}
T_{\varphi z_{k}} T_{\bar{z}_{j}} & =T_{\varphi} T_{\bar{z}_{j}} T_{z_{k}} \\
& =T_{\left(\varphi \diamond \bar{z}_{j}\right) z_{k}} \\
& =T_{\varphi z_{k} \diamond \bar{z}_{j}}, \quad k \neq j \\
T_{\varphi z_{j}} T_{\bar{z}_{j}} & =T_{\varphi}\left(T_{z_{j}} T_{\bar{z}_{j}}\right) \\
& =T_{\varphi}\left(T_{\left|z_{j}\right|^{2}}-2 I\right) \\
& =T_{\varphi} T_{\left|z_{j}\right|^{2}}-T_{2 \varphi} \\
& =T_{\varphi \diamond\left|z_{j}\right|^{2}-2 \varphi} \\
& =T_{\varphi z_{j} \diamond \bar{z}_{j}}, \\
T_{\bar{z}_{k} \varphi} T_{\bar{z}_{j}} & =T_{\bar{z}_{k}}\left(T_{\varphi} T_{\bar{z}_{j}}\right)=T_{\bar{z}_{k}\left(\varphi \diamond \bar{z}_{j}\right)} \\
& =T_{\varphi \bar{z}_{k} \bar{z}_{j}-2 \bar{z}_{k}\left(\partial_{j} \varphi\right)} \\
& =T_{\bar{z}_{k} \varphi \diamond \bar{z}_{j}} .
\end{aligned}
$$

Thus, $T_{\varphi} T_{\bar{z}_{j}}=T_{\varphi \diamond \bar{z}_{j}}$ for all $\varphi$.

Next, for arbitrary $\varphi$ we consider $T_{\varphi} T_{\psi}$ and do induction on the degree of $\psi$. We can assume $\psi$ is monomial. Assume the result for all $\varphi$ and for $\psi$ of fixed degree ( $\psi$ constant is trivial). The inductive step is, first,

$$
T_{\varphi} T_{\psi z_{j}}=\left(T_{\varphi} T_{\psi}\right) T_{z_{j}}=T_{(\varphi \diamond \psi) z_{j}}=T_{\varphi \diamond \psi z_{j}}
$$

We must also consider

$$
T_{\varphi} T_{\bar{z}_{j} \psi}=\left(T_{\varphi} T_{\bar{z}_{j}}\right) T_{\psi}
$$

By the first part of the proof,

$$
T_{\varphi} T_{\bar{z}_{j}}=T_{\varphi \diamond \bar{z}_{j}}
$$

and by the inductive hypothesis

$$
T_{\varphi \diamond \bar{z}_{j}} T_{\psi}=T_{\left(\varphi \diamond \bar{z}_{j}\right) \diamond \psi}
$$

Thus, we need only check that

$$
\varphi \diamond \bar{z}_{j} \psi=\left(\varphi \diamond \bar{z}_{j}\right) \diamond \psi
$$


This is a direct calculation. We note that

$$
\varphi \diamond \bar{z}_{j}=\varphi \bar{z}_{j}-2\left(\partial_{j} \varphi\right)
$$

so

$$
\begin{aligned}
\left(\varphi \diamond \bar{z}_{j}\right) \diamond \psi= & \varphi \bar{z}_{j} \diamond \psi-2\left(\partial_{j} \varphi\right) \diamond \psi \\
= & \sum_{k} \frac{(-2)^{|k|}}{k !} \bar{z}_{j}\left(\partial^{k} \varphi\right)\left(\bar{\partial}^{k} \psi\right) \\
& -2 \sum_{k} \frac{(-2)^{|k|}}{k !}\left(\partial^{k} \partial_{j} \varphi\right)\left(\bar{\partial}^{k} \psi\right) .
\end{aligned}
$$

Using

$$
\bar{\partial}^{k}\left(\bar{z}_{j} \psi\right)=\bar{z}_{j}\left(\bar{\partial}^{k} \psi\right)+k_{j}\left(\bar{\partial}^{k-\delta_{j}} \psi\right)
$$

where

$$
k-\delta_{j}=\left(k_{1}, k_{2}, \ldots, k_{j}-1, k_{j+1}, \ldots, k_{n}\right),
$$

we see that

$$
\begin{aligned}
\varphi \diamond \bar{z}_{j} \psi= & \sum_{k} \frac{(-2)^{|k|}}{k !}\left(\partial^{k} \varphi\right)\left(\bar{\partial}^{k} \bar{z}_{j} \psi\right) \\
= & \sum_{k} \frac{(-2)^{|k|}}{k !} \bar{z}_{j}\left(\partial^{k} \varphi\right)\left(\bar{\partial}^{k} \psi\right) \\
& +\sum_{k} \frac{(-2)^{|k|}}{k !}\left(\partial^{k} \varphi\right) k_{j}\left(\bar{\partial}^{k-\delta_{j}} \psi\right) .
\end{aligned}
$$

Thus, we need only check that

$$
\sum_{k} \frac{(-2)^{|k|}}{k !}\left(\partial^{k} \varphi\right) k_{j}\left(\bar{\partial}^{k-\delta_{j}} \psi\right)=-2 \sum_{k} \frac{(-2)^{|k|}}{k !}\left(\partial^{k} \partial_{j} \varphi\right)\left(\bar{\partial}^{k} \psi\right) .
$$

Reindexing the sum on the left by $\ell=k-\delta_{j}$ completes the proof.

Remark. Since $\bar{z}_{j} \diamond \psi=\bar{z}_{j} \psi$, the identity

$$
\varphi \diamond \bar{z}_{j} \psi=\left(\varphi \diamond \bar{z}_{j}\right) \diamond \psi
$$

follows from the reasonably well-known associativity of $\diamond[\mathrm{G}]$. Our computational proof has the advantage of giving associativity of $\diamond$ as an immediate corollary of Theorem 2 since

$$
\begin{gathered}
T_{\varphi \diamond(\psi \diamond \gamma)}=T_{\varphi}\left(T_{\psi} T_{\gamma}\right)=\left(T_{\varphi} T_{\psi}\right) T_{\gamma}=T_{(\varphi \diamond \psi) \diamond \gamma} \\
\text { 3. } T_{\varphi} \text { WITH } T_{\varphi} T_{\varphi} \neq T_{\psi} \text { FOR ANY } \psi
\end{gathered}
$$

In this section, we produce the promised obstruction to composition of BerezinToeplitz operators. We use some calculations from [BC2] and we begin with a needed improvement of $\left[\mathrm{BC} 2\right.$, Theorem 17]. In this section, we work on $H^{2}(\mathbf{C}, d \mu)$ $(n=1)$. Here, the Bergman reproducing kernel function for evaluation at $z$ is just

$$
K(w, z)=e^{w \bar{z} / 2}
$$

and it follows that

$$
k_{z}(w)=K(w, z) / \sqrt{K(z, z)}=e^{w \bar{z} / 2-|z|^{2} / 4}
$$


is a unit vector in $H^{2}(\mathbf{C}, d \mu)$. We consider the unitary operator

$$
\left(R_{a} f\right)(z)=f(a z)
$$

on $H^{2}(\mathbf{C}, d \mu)$ for $|a|=1$.

Theorem 3. For $|a|=1$ and $\operatorname{Re} a<0$, we have

$$
\left\|R_{a}-T_{\psi}\right\| \geq 1
$$

for all $\psi$ such that $\psi K(\cdot, z)$ is in $L^{2}(\mathbf{C}, d \mu)$ for every $z$ in $\mathbf{C}$.

Proof. We consider

$$
\begin{aligned}
\left\|T_{\psi}-R_{a}\right\| & \geq\left|\left\langle T_{\psi} k_{z}, R_{a} k_{z}\right\rangle-\left\langle R_{a} k_{z}, R_{a} k_{z}\right\rangle\right| \\
& \geq\left|\left\langle T_{\psi} k_{z}, R_{a} k_{z}\right\rangle-1\right| .
\end{aligned}
$$

Now,

$$
\left\langle T_{\psi} k_{z}, R_{a} k_{z}\right\rangle=\left\langle\psi \chi_{z}, K(\cdot,(1+\bar{a}) z)\right\rangle e^{-|z|^{2} / 2}
$$

so we have

$$
\begin{aligned}
\left|\left\langle T_{\psi} k_{z}, R_{a} k_{z}\right\rangle\right| & \leq e^{-|z|^{2} / 2}\|\psi\| \sqrt{K((1+\bar{a}) z,(1+\bar{a}) z)} \\
& \leq\|\psi\| e^{-|z|^{2} / 2} e^{|1+a|^{2}|z|^{2} / 4} \\
& \leq\|\psi\| e^{|z|^{2} \operatorname{Re} a / 2}
\end{aligned}
$$

Since Re $a<0$, we see that

$$
\left|\left\langle T_{\psi} k_{z}, R_{a} k_{z}\right\rangle\right| \rightarrow 0
$$

as $|z| \rightarrow \infty$. Thus, $\left\|T_{\psi}-R_{a}\right\| \geq 1$.

The function $\varphi$ will be chosen to have the form $\varphi(z)=e^{\lambda|z|^{2}}$ where $\operatorname{Re} \lambda<\frac{1}{4}$ so that $T_{\varphi}$ makes sense.

Lemma. For $\lambda=\frac{1}{5}+i \frac{2}{5}$ and $\varphi(z)=e^{\lambda|z|^{2}}$, we have $T_{\varphi}$ unitary with

$$
T_{\varphi} T_{\varphi}=a R_{a}
$$

for $a=\overline{(1-2 \lambda)^{2}}=-\frac{7}{25}+i \frac{24}{25}$.

Proof. $\operatorname{Re} \lambda<\frac{1}{4}$ and calculations outlined in [BC2, p. 582] show that $T_{\varphi}$ is diagonal in the basis

$$
e_{k}=\left(2^{k} k !\right)^{-1 / 2} z^{k}, \quad k=0,1, \ldots
$$

for $H^{2}(\mathbf{C}, d \mu)$, with

$$
T_{\varphi} e_{k}=(1-2 \lambda)^{-(k+1)} e_{k}
$$

Now

$$
\lambda=\frac{1}{5}+i \frac{2}{5}
$$

and so

$$
\begin{aligned}
T_{\varphi} T_{\varphi} e_{k} & =\overline{(1-2 \lambda)^{2(k+1)}} e_{k} \\
& =a^{k+1} e_{k} .
\end{aligned}
$$

But

$$
a R_{a} e_{k}=a^{k+1} e_{k}
$$

and we are done. 
We now have the promised

Theorem 4. For $\lambda=\frac{1}{5}+i \frac{2}{5}$ and $a=\overline{(1-2 \lambda)^{2}}=-\frac{7}{25}+\frac{24}{25} i$, with $\varphi(z)=e^{\lambda|z|^{2}}$,

$$
\left\|T_{\varphi} T_{\varphi}-T_{\psi}\right\| \geq 1
$$

for $\underline{\text { all }} \psi$ such that $\psi K(\cdot, z)$ is in $L^{2}(\mathbf{C}, d \mu)$ for every $z$ in $\mathbf{C}$.

Proof. Direct combination of Theorem 3 and the Lemma.

Remark. In fact, for $\varphi(z)=e^{\lambda|z|^{2}},(* *)$ yields

$$
\varphi \diamond \varphi=e^{\mu|z|^{2}}
$$

where $\mu=2 \lambda(1-\lambda)$. Thus, for $\lambda=\frac{1}{5}+i \frac{2}{5}$, we have $\mu=\frac{16}{25}+i \frac{12}{25}$ and $e^{\mu|z|^{2}} f(z)$ cannot be in $L^{2}(\mathbf{C}, d \mu)$ for any $f \neq 0$ in $H^{2}(\mathbf{C}, d \mu)$.

\section{REMARKS}

There is a considerable space between Theorems 1 and 2 and Theorem 4 . It does not seem easy to lift the known much stronger positive results directly over from the setting of pseudodifferential operators. It does seem likely that $(* *)$ provides a composition formula for Berezin-Toeplitz operators in a setting substantially larger than those of Theorems 1 and 2. For non- $C^{\infty} \varphi, \psi$ or even for general $C^{\infty} \varphi, \psi$, the problem of determining whether there is a $\varphi \diamond \psi$ with $T_{\varphi} T_{\psi}=T_{\varphi \diamond \psi}$, as well as the form of $\varphi \diamond \psi$, remains open.

Theorems 1 and 2 can be extended to the natural family of Gaussian measures on $\mathbf{C}^{n}$ which provide representation spaces for the Heisenberg group [C]. For $d \mu_{r}(z)=$ $\left(\frac{r}{\pi}\right)^{n} e^{-r|z|^{2}} d v(z)$ with $r>0$ and $H^{2}\left(\mathbf{C}^{n}, d \mu_{r}\right)$ as before, we have Bergman kernels

$$
K_{r}(w, z)=e^{r w \cdot z}
$$

and Berezin-Toeplitz operators on $H^{2}\left(\mathbf{C}^{n}, d \mu_{r}\right)$

$$
\left(T_{\varphi}^{(r)} f\right)(z)=\int_{\mathbf{C}^{n}} e^{r z \cdot w} \varphi(w) f(w) d \mu_{r}(w) .
$$

Then minor modifications yield

Theorem 1'. For $\varphi, \psi$ in $B_{a}\left(\mathbf{C}^{n}\right), \varphi \diamond_{r} \psi$ is also in $B_{a}\left(\mathbf{C}^{n}\right)$ for

$$
\varphi \diamond_{r} \psi=\sum_{k}\left(\frac{-1}{r}\right)^{|k|} \frac{1}{k !}\left(\partial^{k} \varphi\right)\left(\bar{\partial}^{k} \psi\right)
$$

and $T_{\varphi}^{(r)} T_{\psi}^{(r)}=T_{\varphi \diamond_{r} \psi}^{(r)}$. The series in $(\dagger)$ converges uniformly and absolutely. Moreover, for $r>1$

$$
\left\|\varphi \diamond_{r} \psi-\sum_{|k| \leq K}\left(\frac{-1}{r}\right)^{|k|} \frac{1}{k !}\left(\partial^{k} \varphi\right)\left(\bar{\partial}^{k} \psi\right)\right\|_{\infty} \leq \frac{1}{r^{K+1}} C(\varphi, \psi, K)
$$

for $C(\varphi, \psi, K)$ a constant independent of $r$.

Theorem $2^{\prime}$. For $\varphi, \psi$ polynomials in $\left(z_{1}, \ldots, z_{n}, \bar{z}_{1}, \ldots, \bar{z}_{n}\right)$, we have $T_{\varphi}^{(r)} T_{\psi}^{(r)}$ defined on a dense domain consisting of linear combinations of functions of the form $\left\{p(z) e^{z \cdot a}: a \in \mathbf{C}^{n}\right.$ and $p(z)$ polynomial in $\left.\left(z_{1}, \ldots, z_{n}\right)\right\}$. On this domain

$$
T_{\varphi}^{(r)} T_{\psi}^{(r)}=T_{\varphi \diamond_{r} \psi}^{(r)}
$$

for $\varphi \diamond_{r} \psi$ given by $(\dagger)$ and $\varphi \diamond_{r} \psi$ is polynomial in the $z_{j}, \bar{z}_{j}$. 
While Theorems 1 and 2 provide some basis for optimism about the development of a reasonably extensive Berezin-Toeplitz calculus on $\mathbf{C}^{n}$, the situation is considerably less promising on the classical Bergman space of the disc, $H^{2}\left(\mathbf{D}, \frac{d A}{\pi}\right)$, where $\mathbf{D}=\{z \in \mathbf{C}:|z|<1\}$ and $\frac{d A}{\pi}$ is normalized Lebesgue area measure. In this case, the Bergman kernel function is just $K(z, w)=(1-z \bar{w})^{-2}$ and the Berezin-Toeplitz operator $T_{\varphi}$ on $H^{2}\left(\mathbf{D}, \frac{d A}{\pi}\right)$ is given by

$$
\left(T_{\varphi} f\right)(z)=\int_{\mathbf{D}} K(z, w) \varphi(w) f(w) \frac{d A(w)}{\pi} .
$$

Direct calculation shows, first, that

$$
T_{z} T_{\bar{z}}=T_{1+\log |z|^{2}} .
$$

Moreover,

$$
T_{z^{2}} T_{\bar{z}^{2}}=T_{1+2 \log |z|^{2}}+P_{0}
$$

where $P_{0} f=\int_{\mathbf{D}} f(z) \frac{d A(z)}{\pi}$ and $P_{0} \neq T_{\varphi}$ for any $\varphi$. For asymptotic results on composition of Berezin-Toeplitz operators on $H^{2}\left(\mathbf{D}, \frac{d A}{\pi}\right)$ see [KL].

\section{REFERENCES}

[B] V. Bargmann, On a Hilbert space of analytic functions and an associated integral transform, Comm. Pure and Appl. Math. 14 (1961), 187-214. MR 28:486

[Be] F. A. Berezin, Covariant and contravariant symbols of operators, Izv. Akad. Nauk SSSR Ser. Mat. 36 (1972), 1134-1167. MR 50:2996

[BC1] C. A. Berger and L. A. Coburn, Toeplitz operators on the Segal-Bargmann space, Trans. AMS 301 (1987), 813-829. MR 88c:47044

[BC2] Heat flow and Berezin-Toeplitz estimates, Amer. J. Math. 116 (1994), 563-590. MR 95g:47038

[C] L. A. Coburn, The measure algebra of the Heisenberg group, J. Funct. Analysis 161 (1999), 509-525. MR 2000b:46128

[F] G. B. Folland, Harmonic analysis in phase space, Annals of Math. Studies, Princeton Univ. Press, Princeton, N.J., 1989. MR 92k:22017

[G] M. Gerstenhaber, On the deformation of rings and algebras, III, Annals. of Math. (2) 88 (1968), 1-34. MR 39:1521

[Gu V. Guillemin, Toeplitz operators in n-dimensions, Integral Equations and Operator Theory 7 (1984), 145-205. MR 86i:58130

[KL] S. Klimek and A. Lesniewski, Quantum Riemann surfaces, I. The unit disc, Comm. Math. Phys. 146 (1992) 103-122. MR 93g:58009

Department of Mathematics, State University of New York at Buffalo, Buffalo, New YoRK 14260

E-mail address: lcoburn@acsu.buffalo.edu 